\title{
A Note on Fuzzy Relational Matrices
}

\author{
Mamoni Dhar \\ Assistant Professor, Department of Mathematics, Science College, Kokrajhar, Assam, India \\ E-mail:mamonidhar@rediffmail.com/mamonidhar@gmail.com
}

\begin{abstract}
In this article, we would like to revisit the existing definitions of cartesian product of fuzzy sets and the fuzzy relational matrix from the standpoint of the new definition of fuzzy sets on the basis of reference function. In the process it is observed that there are drawbacks in the current defintions and hence new definitions of terms mentioned are proposed in this article. Some numerical examples are also presented to make the matter clear and simple.
\end{abstract}

Index Terms - Reference Function, Membership Value, Closed Expressions, Table Searching

\section{Introduction}

Since fuzzy set theory proposed by Zadeh [1], it has been developed in theory and applications in the past 45 years. One such application area can be found in Zadeh [2]. Fuzzy relation, a basic approach for presenting propositions can be used to describe various relations of various elements. These elements refer to fuzzy objects, fuzzy events, fuzzy properties and fuzzy vectors and so on. How to confirm the membership of fuzzy relation is also a problem. The general approach includes cartesian product, closed expression, table searching, lingual rule of knowledge, classification, digital rule of knowledge, similarity approaches in digital knowledge.

In this article, we would like to stess on the use of reference function in the representation of fuzzy sets. Thereafter, we shall deal with fuzzy relational matrices which are the outcome of the cartesian products of fuzzy sets.

The main contribution of this article is to present a new definition of cartesian product of fuzzy sets when fuzzy sets are represented in terms of reference function and afterwards a new method of representation of fuzzy relational matrices is introduced.

Finally, we shall prove some properties of fuzzy relational matrix and use several examples to compare the proposed catesian product of two fuzzy sets with the existing method. Numerical result shows that the proposed method is similar to the existing one except in cases of representation of the elements of the matrix so obtained.

The remainder of the paper is organized as follows: In Section II, some important definitions on which we would like to comment is presented.Section III deals with Baruah's definition of fuzzy sets on the basis of reference function. Section IV presents the new definition of cartesian product of fuzzy sets. Section V presents the newly introduced definition of fuzzy relational matrix. In section VI, some properties of fuzzy relational matrix are discussed and finally, Section VII presents the conclusions.

Before proceeding further with the definition of fuzzy relational matrices, let us have a look at the definitions of the Cartesian products and fuzzy relation in the following section.

\section{Some Important Definitions}

This section deals with the existing definitions of cartesian product of fuzzy sets and fuzzy relational matrix.

\subsection{Cartesian Products of Fuzzy Sets}

Let $A_{1}, A_{2}, \ldots \ldots \ldots, A_{n}$ be fuzzy sets in $\Omega_{1}, \Omega_{2}, \ldots \ldots \ldots, \Omega_{n}$ respectively. The cartesian product of $A_{1}, A_{2}, \ldots \ldots \ldots, A_{n}$ is fuzzy set in the space $\Omega_{1} \times \Omega_{2} \times \ldots \ldots \ldots \times \Omega_{n}$ with membership function as:

$$
\begin{aligned}
& \mu_{A_{1} \times A_{2} \times \ldots \ldots . \times A_{n}}\left(x_{1}, x_{2}, \ldots \ldots . ., x_{n}\right) \\
= & \min \left(\mu_{A}\left(x_{1}\right), \mu_{A}\left(x_{2}\right), \ldots \ldots, \mu_{A}\left(x_{1}\right)\right)
\end{aligned}
$$

So, the cartesian product of $A_{1}, A_{2}, \ldots \ldots \ldots, A_{n}$ is denoted by $A_{1} \times A_{2} \times \ldots \ldots \ldots \times A_{n}$

\section{Numerical Example:} $0.6)\}$

Let $\mathrm{A}=\{(3,0.5),(5,1),(7,0.6)\}$ and $\mathrm{B}=\{(3,1),(5$,

The product is the all set of pairs with minimum associated memberships.

$$
\begin{aligned}
A \times B=\{[(3,3), 0.5],[(3,5), 0.5],[(5,3), 1],[(7,3), 0.6], & \\
& {[(5,5), 0.6],[(7,3), 0.6],[(7,5), 0.6]\} }
\end{aligned}
$$




\subsection{Fuzzy Relations}

The concept of fuzzy relation is a natural extension to fuzzy sets and plays an important role in the theory of such sets and their applications. A fuzzy relation $\mathrm{R}$ from a fuzzy set $\mathrm{A}$ in $\mathrm{X}$ to a fuzzy set $\mathrm{B}$ in $\mathrm{Y}$ is a fuzzy set which is the cartesian product $A \times B$ in the cartesian product space $X \times Y$. $\mathrm{R}$ is characterized by the membership function expressing various degrees of relations as

$$
R=A \times B=\sum\left(\mu_{A}(x), \mu_{B}(y)\right)
$$

It is to be noted here that the sum does not mean a mathematical summation operation; it means all possible combination of all elements. $\mathrm{R}$ is also called the relational matrix.

$$
R=A \times B=\left(\begin{array}{cc}
0.5 & 0.5 \\
1 & 0.6 \\
0.6 & 0.6
\end{array}\right)
$$

It is important to mention here that we are interested in dealing with fuzzy sets in terms of reference function because it plays a very important role in case of complementation of fuzzy sets. It is seen that very often the cartesian product is found which involves complementation of fuzzy sets and so there is the need of defining cartesian product accordingly.

\section{Baruah's Definition of Complementation of Fuzzy Sets}

Baruah([3],[4]\&[5] )has defined a fuzzy number $\mathrm{N}$ with the help of two functions :a fuzzy membership function $\mu_{2}(x)$ and a reference function $\mu_{1}(x)$ such that $0 \leq \mu_{1}(x) \leq \mu_{2}(x) \leq 1$ Then for a fuzzy number denoted by $\left\{x, \mu_{2}(x), \mu_{1}(x), x \in \Omega\right\}$ we would call $\mu_{2}(x)-\mu_{1}(x)$ as the fuzzy membership value ,which is different from fuzzy membership function .It is to be noted here that in the definition of complement of a fuzzy set, fuzzy membership value and the fuzzy membership function have to be different, in the sense that for a usual fuzzy set the membership value and membership function are of course equivalent.

In accordance with the process discussed above, a fuzzy set defined by

$$
A=\{x, \mu(x), x \in \Omega\}
$$

would be defined in this way as

$$
A=\{x, \mu(x), 0, x \in \Omega\}
$$

So that the complement would become

$$
A^{c}=\{x, 1, \mu(x), x \in \Omega\}
$$

This definition plays a key role in dealing with cases which invole especially the complementation of fuzzy sets. This result has been applied in various other cases of fuzzy sets, Dhar ( [6],[7],[8],[9],[10],[11],[12],[13],[14]).

Now in the following sections, we would like to present the new definitions of cartesian product of two fuzzy sets and the fuzzy relational matrix so obtained. It is expected that the new results will help in bridging the gap that currently exists in the current literature.

\section{New Definition of Cartesian Product of Fuzzy Sets}

Let $A_{1}, A_{2}, \ldots \ldots \ldots, A_{n}$ be fuzzy sets defined in terms of reference function in $\Omega_{1}, \Omega_{2}, \ldots \ldots \ldots, \Omega_{n}$ respectively. The cartesian product of $A_{1}, A_{2}, \ldots \ldots \ldots, A_{n}$ is fuzzy set in the space $\Omega_{1} \times \Omega_{2} \times \ldots \ldots \ldots \times \Omega_{n}$ which would have to be defined with membership function as:

$$
\begin{aligned}
& \mu_{A_{1} \times A_{2} \times \ldots \ldots . \times A_{n}}\left(x_{1}, x_{2}, \ldots \ldots . ., x_{n}\right) \\
= & \min \left(\mu_{A}\left(x_{1}\right), \mu_{A}\left(x_{2}\right), \ldots \ldots, \mu_{A}\left(x_{n}\right)\right), \\
& \max \left(\mu_{A}^{\prime}\left(x_{1}\right), \mu_{A}^{\prime}\left(x_{2}\right), \ldots \ldots, \mu_{A}^{\prime}\left(x_{n}\right)\right\}
\end{aligned}
$$

Where $\mu_{A}^{\prime}\left(x_{1}\right), \mu_{A}^{\prime}\left(x_{2}\right), \ldots \ldots, \mu_{A}^{\prime}\left(x_{n}\right)$ are the reference functions of the fuzzy sets $A_{1}, A_{2}, \ldots \ldots \ldots, A_{n}$

We would like to discuss it in the following way

Let us consider two fuzzy sets expressed according to the new definition as

$$
A=\left\{u_{i}, \mu_{A}\left(u_{i}\right), \mu_{A^{\prime}}\left(u_{i}\right)\right\}
$$

and

$$
B=\left\{v_{i}, \mu_{B}\left(v_{i}\right), \mu_{B^{\prime}}\left(v_{i}\right)\right\}
$$

Then their cartesian product would be presented as 


$$
A \times B=\left(\begin{array}{l}
\left(\mu_{A}\left(u_{1}\right), \mu_{A^{\prime}}\left(u_{1}\right)\right) \\
\left(\mu_{A}\left(u_{2}\right), \mu_{A^{\prime}}\left(u_{2}\right)\right. \\
\cdot \\
\cdot \\
\cdot \\
\left(\mu_{A}\left(u_{n}\right), \mu_{A^{\prime}}\left(u_{n}\right)\right)
\end{array}\right) \times\left(\mu_{B}\left(v_{1}\right), \mu_{B^{\prime}}\left(v_{1}\right)\right) \quad\left(\mu_{B}\left(v_{2}\right), \mu_{B^{\prime}}\left(v_{2}\right)\right) \ldots \quad\left(\mu_{B}\left(v_{n}\right), \mu_{B^{\prime}}\left(v_{n}\right)\right)
$$

Let us consider

$$
A \times B=\left(\begin{array}{lll} 
& & \\
C_{11} & C_{12} \ldots \ldots . & C_{1 n} \\
C_{21} & C_{22} \ldots \ldots . & C_{2 n} \\
\ldots & \ldots & \ldots \\
C_{n 1} & C_{n 2} \ldots \ldots . & C_{n n}
\end{array}\right)
$$

where

$$
\begin{aligned}
& C_{11}=\left[\min \left\{\mu_{A}\left(u_{1}\right), \mu_{B}\left(v_{1}\right)\right\}, \max \left\{\left(\mu_{A^{\prime}}\left(u_{1}\right), \mu_{B^{\prime}}\left(v_{1}\right)\right\}\right]\right. \\
& C_{12}=\left[\min \left\{\mu_{A}\left(u_{1}\right), \mu_{B}\left(v_{2}\right)\right\}, \max \left\{\left(\mu_{A^{\prime}}\left(u_{1}\right), \mu_{B^{\prime}}\left(v_{2}\right)\right\}\right]\right.
\end{aligned}
$$

$C_{1 n}=\left[\min \left\{\mu_{A}\left(u_{1}\right), \mu_{B}\left(v_{n}\right)\right\}, \max \left\{\left(\mu_{A^{\prime}}\left(u_{1}\right), \mu_{B^{\prime}}\left(v_{n}\right)\right\}\right]\right.$

and similar for others.

For usual fuzzy sets we are to consider

$$
\mu_{A^{\prime}}\left(u_{1}\right)=\mu_{A^{\prime}}\left(u_{2}\right)=\ldots \ldots \ldots \ldots \ldots=\mu_{A^{\prime}}\left(u_{n}\right)=0
$$

and also

$$
\mu_{B^{\prime}}\left(v_{1}\right)=\mu_{B^{\prime}}\left(v_{2}\right)=\ldots \ldots \ldots \ldots \ldots=\mu_{B^{\prime}}\left(v_{n}\right)=0
$$

Now let us consider the cartesian product $A^{c} \times B$ of two fuzzy sets $A^{c}$ and $B$ in the following way

$$
A^{c} \times B=\left(\begin{array}{lll}
D_{11} & D_{12} \ldots \ldots & D_{1 n} \\
D_{21} & D_{22} \ldots \ldots . & D_{2 n} \\
\cdots & \cdots & \cdots \\
D_{n 1} & D_{n 2} \ldots \ldots . & D_{n n}
\end{array}\right)
$$

Where

$$
\begin{aligned}
D_{11} & =\left[\min \left\{1, \mu_{B}\left(v_{1}\right)\right\}, \max \left\{\left(\mu_{A}\left(u_{1}\right), 0\right\}\right]\right. \\
& =\left(\mu_{B}\left(v_{1}\right), \mu_{A}\left(u_{1}\right)\right), \operatorname{provided} \mu_{B}\left(v_{1}\right) \geq \mu_{A}\left(u_{1}\right)
\end{aligned}
$$

$$
\begin{aligned}
D_{12} & =\left[\min \left\{1, \mu_{B}\left(v_{2}\right)\right\}, \max \left\{\left(\mu_{A}\left(u_{1}\right), 0\right\}\right]\right. \\
& =\left(\mu_{B}\left(v_{2}\right), \mu_{A}\left(u_{1}\right)\right), \text { provided } \mu_{B}\left(v_{2}\right) \geq \mu_{A}\left(u_{1}\right)
\end{aligned}
$$

$$
D_{1 n}=\left[\min \left\{1, \mu_{B}\left(v_{n}\right)\right\}, \max \left\{\left(\mu_{A}\left(u_{1}\right), 0\right\}\right]\right.
$$$$
=\left(\mu_{B}\left(v_{n}\right), \mu_{A}\left(u_{1}\right)\right) \text {, provided } \mu_{B}\left(v_{n}\right) \geq \mu_{A}\left(u_{1}\right)
$$

and so on .

It is important to note here that using the reference function, here we have introduced a method of calculating the cartesian product of two fuzzy sets $A^{c}$ and $B$ but for this we have considered $\mu_{A^{C}}\left(u_{i}\right) \geq \mu_{B}\left(v_{i}\right)$.

Similarly if we find the cartesian product $B^{c} \times A$, then

$$
\mu_{B^{c}}\left(v_{i}\right) \geq \mu_{A}\left(u_{i}\right)
$$

For other cases, we are trying to find the resutls .

A numerical example is presented below to make our point clear.

\section{Numerical Example:}

The fuzzy sets $A=\{(3,0.5),(5,1),(7,0.6)\}$, and $\mathrm{B}=\{(3,1),(5,0.6)\}$, would be represented in our way as

$$
A=\{(3,0.5,0),(5,1,0),(7,0.6,0)\}
$$

and

$$
\mathrm{B}=\{(3,1,0),(5,0.6,0)\}
$$

Then the cartesian product would have to be calculated in the following way

$A \times B=\{[(3,3), \min (0.5,1), \max (0,0)],[(3,5), \min (0.5,0.6$ )$], \max (0,0)],[(5,3), \min (1,1), \max (0.0)],[(5,5), \min (0.6,1)$, $\max (0,0)],[(7,3), \min (0.6,1), \max (0,0)]$, $[(7,5), \min (0.6,1), \max (0,0)]\}$ 
$=\{[(3,3),(0.5,0)]$,

$[(3,5),(0.5,0)],[(5,3),(1,0)],[(5,5),(0.6,0)],[(7,3),(0.6,0)]$,

$$
[(7,5),(0.6,0)]\}
$$

\section{New Definition of Relational Matrix}

The cartesian product $R=A \times B$ represented on the basis of reference functions which composes a relation between fuzzy sets A and B is called fuzzy relational matrix.

In the above case fuzzy relational matrix will take the following form:

$$
R=A \times B=\left(\begin{array}{cc}
(0.5,0) & (0.5,0) \\
(1,0) & (0.6,0) \\
(0.6,0) & (0.6,0)
\end{array}\right)
$$

According to the new definition of complementation of fuzzy sets, we get the complement of the cartesian product $A \times B$ as

$$
\begin{aligned}
& A \times B=\{[(3,3),(1,0.5)],[3,5),(1,0.5)],[(5,3),(1,1)], \\
& [(5,5),(1,0.6)],[(7,3),(1,0.6)],[(7,5),(1,0.6)]\}
\end{aligned}
$$

In this case, the relational matrix will take the form

$$
R^{c}=(A \times B)^{c}=\left(\begin{array}{cc}
(1,0.5) & (1,0.5) \\
(1,1) & (1,0.6) \\
(1,0.6) & (1,0.6)
\end{array}\right)
$$

\section{Properties of Fuzzy Relational Matrix}

In this section, we shall discuss some properties of relational matrix of fuzzy sets. It is important to note here that when finding the composition of relational matrices of fuzzy sets is straightforward like matrix multiplication .Let us have a brief look at the multiplication of fuzzy relational matrices

The multiplication of two fuzzy relational matrices A and $\mathrm{B}$ which are conformable for multiplication will be defined in the following manner:

$$
A B=\left\{\max \min \left(a_{i j}, b_{j i}\right), \min \max \left(r_{i j}, r_{j i}^{\prime}\right)\right\}
$$

It is important to note that while finding the min-max composition of two fuzzy relational matrices, we are to replace maxmin by minmax and minmax by maxmin in the above formula of multiplication.

Where $a_{i j}$ stands for the membership function of the fuzzy matrix A for the ith row and jth column and $r_{i j}$ is the corresponding reference function and $b_{i j}$ stands for the membership function of the fuzzy matrix $B$ for the ith row and jth column where $r_{i j}^{\prime}$ represents the corresponding reference function.

\section{Property1}

It can be easily verified with examples of two fuzzy relational matrices which are conformable, the following result holds good

$$
\left(R_{1} o R_{2}\right)^{c}=R_{1}^{c} \Phi R_{2}^{c}
$$

Where $R_{1}$ and $R_{2}$ are two relational matrices, c denotes one's complement operation over the elements of the matrix, o and $\Phi$ denote max-min and min-max composition operator respectively.

It is important to note here that the above mentioned properties hold for both existing method and for the newly defined relational matrices.

\section{Numerical Example}

Let us consider two relational matrices as

$$
\begin{aligned}
& R_{1}=\left(\begin{array}{cc}
(0.5,0) & (0.5,0) \\
(1,0) & (0.6,0) \\
(0.6,0) & (0.6,0)
\end{array}\right) \text { and } \\
& R_{2}=\left(\begin{array}{lll}
(0.2,0) & (0.5,0) & (0.4,0) \\
(0.2,0) & (0.6,0) & (0.4,0)
\end{array}\right)
\end{aligned}
$$

Then

$$
\begin{aligned}
R_{1} o R_{2}= & \left(\begin{array}{cc}
(0.5,0) & (0.5,0) \\
(1,0) & (0.6,0) \\
(0.6,0) & (0.6,0)
\end{array}\right) \\
& o\left(\begin{array}{lll}
(0.2,0) & (0.5,0) & (0.4,0) \\
(0.2,0) & (0.6,0) & (0.4,0)
\end{array}\right)
\end{aligned}
$$

Let us suppose that

$$
R_{1} o R_{2}=\left(\begin{array}{lll}
r_{11} & r_{12} & r_{13} \\
r_{21} & r_{22} & r_{23} \\
r_{31} & r_{32} & r_{33}
\end{array}\right)
$$

where

$$
r_{11}=[\max \{\min (0.5,0.2), \min (0.5,0.2)\}, \min \{\max
$$
$(0.0), \operatorname{Max}(0.0)\}]=(0.2,0)$ 


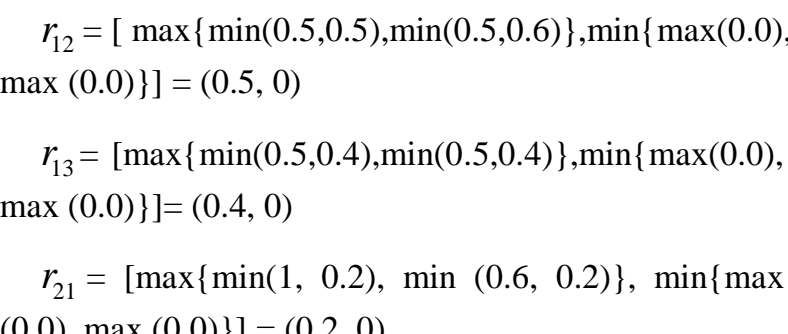
$(0.0), \max (0.0)\}]=(0.2,0)$

$r_{22}=[\max \{\min (1,0.5), \min (0.6,0.6)\}, \min \{\max (0.0)$, $\max (0.0)\}]=(0.6,0)$

$r_{23}=[\max \{\min (1,0.4), \min (0.6,0.4)\}, \min \{\max$ $(0.0), \max (0.0)\}]=(0.4,0)$

$r_{31}=[\max \{\min (0.6,0.2), \min (0.6,0.2)\}, \min \{\max$ $(0.0), \max (0.0)\}]=(0.2,0)$

$r_{32}=[\max \{\min (0.6,0.5), \min (0.6,0.6)\}, \min \{\max$ $(0.0), \max (0.0)\}]=(0.6,0)$

$r_{33}=[\max \{\min (0.6,0.4), \min (0.6,0.4)\}, \min \{\max (0.0)$, $\operatorname{Max}(0.0)\}]=(0.4,0)$

Hence, we get

$$
R_{1} o R_{2}=\left(\begin{array}{lll}
(0.2,0) & (0.5,0) & (0.4,0) \\
(0.2,0) & (0.6,0) & (0.4,0) \\
(0.2,0) & (0.6,0) & (0.4,0)
\end{array}\right)
$$

The elements of the above relation are fuzzy sets and so in case of complementation of the above relation; we would like to use the new definition of complementtion of fuzzy sets to make it logical.

Then the complement of the above relational matrix would have to be written as

$$
\left(R_{1} o R_{2}\right)^{c}=\left(\begin{array}{lll}
(1,0.2) & (1,0.5) & (1,0.4) \\
(1,0.2) & (1,0.6) & (1,0.4) \\
(1,0.2) & (1,0.6) & (1,0.4)
\end{array}\right)
$$

Now proceeding similarly we get

$$
\begin{aligned}
R_{1}^{c} o R_{2}^{c} & =\left(\begin{array}{cc}
(1,0.5) & (1,0.5) \\
(1,1) & (1,0.6) \\
(1,0.6) & (1,0.6)
\end{array}\right) \\
& o\left(\begin{array}{lll}
(1,0.2) & (1,0.5) & (1,0.4) \\
(1,0.2) & (1,0.6) & (1,0.4)
\end{array}\right)
\end{aligned}
$$

$$
=\left(\begin{array}{lll}
(1,0.2) & (1,0.5) & (1,0.4) \\
(1,0.2) & (1,0.6) & (1,0.4) \\
(1,0.2) & (1,0.6) & (1,0.4)
\end{array}\right)
$$

Hence is our claim.

\section{Property2}

If $\mathrm{R}$ be any fuzzy relation then the following property holds

$$
\operatorname{RoR}^{c} \neq \text { nullrelation }
$$

\section{Numerical Example}

Let us consider a fuzzy relation $\mathrm{R}$ and its complementrelation $R^{c}$ in the following way:

$$
\begin{aligned}
& R=\left(\begin{array}{ccc}
(0.5,0) & (0.5,0) & (0.4,0) \\
(1,0) & (0.6,0) & (0.3,0) \\
(0.6,0) & (0.6,0) & (0.5,0)
\end{array}\right) \text { and } \\
& R^{c}=\left(\begin{array}{ccc}
(1,0.5) & (1,0.5) & (1,0.4) \\
(1,1) & (1,0.6) & (1,0.3) \\
(1,0.6) & (1,0.6) & (1,0.5)
\end{array}\right)
\end{aligned}
$$

Proceeding as above we get

$$
\operatorname{RoR}^{c}=\left(\begin{array}{lll}
C_{11} & C_{12} & C_{13} \\
C_{21} & C_{22} & C_{23} \\
C_{31} & C_{32} & C_{33}
\end{array}\right)
$$

Now we get the following:

$r_{11}=[\max \{\min (0.5,1), \min (0.5,1), \min (0.4,1)\}$, $\min \{\max (0,0.5), \max (0,1), \max (0,0.6)\}]$

$=[\max (0.5,0.5$, and 0.4$), \min (0.5,1$, and 0.6$)]$

$=(0.5,0.5)$

$r_{12}=[\max \{\min (0.5,1), \min (0.5,1), \min (0.4,1)\}$, $\min \{\max (0.0 .5), \max (0.0 .6), \max (0,0.6\}]$

$=(0.5,0.5)$

$r_{13}=[\max \{\min (0.5,1), \min (0.5,1), \min (0.4,1)\}$, $\min \{\max (0.0 .4), \max (0.0 .3), \max (0,0.5)\}]$

$=(0.5,0.3)$ 


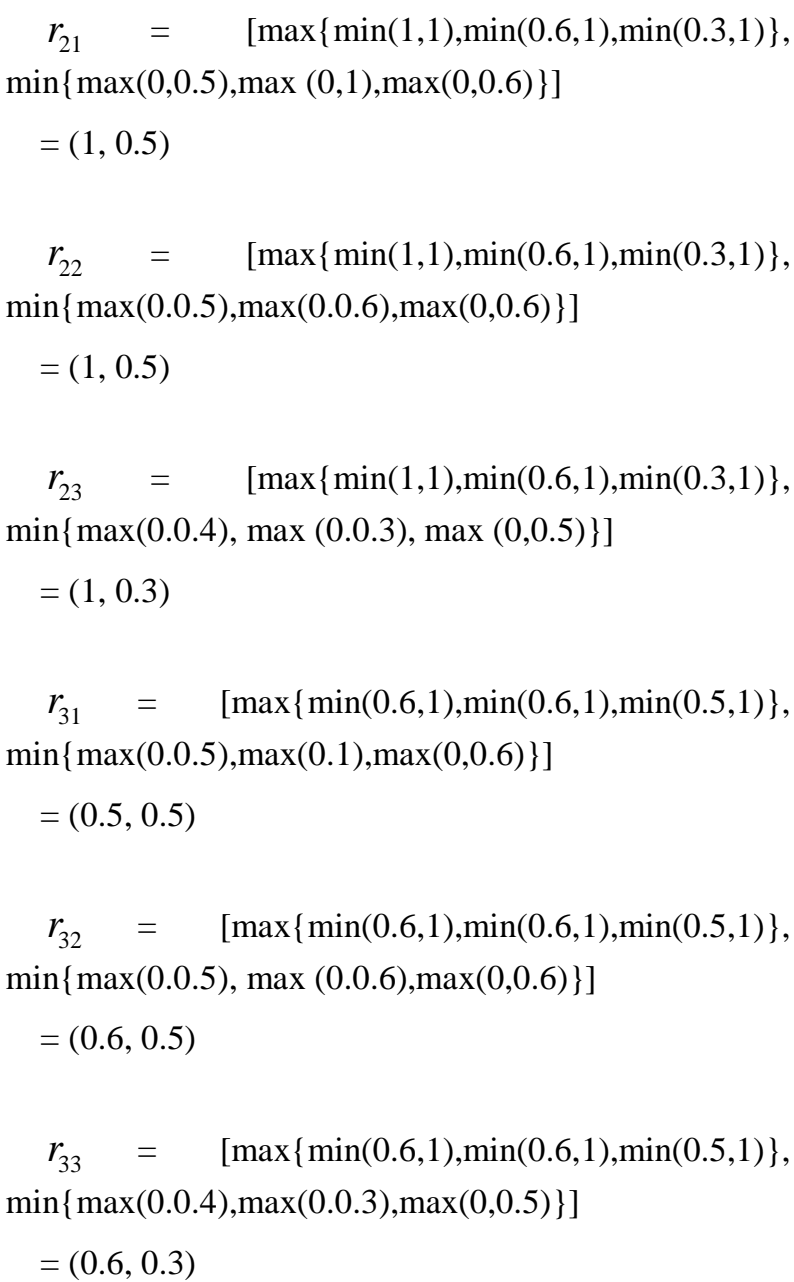

The above result shows that

$$
\begin{aligned}
& \operatorname{RoR}^{c}= \\
& \qquad\left(\begin{array}{ccc}
(0.5,0.5) & (0.5,0.5) & (0.5,0.3) \\
(1,0.5) & (1,0.5) & (1,0.3) \\
(0.5,0.5) & (0.6,0.5) & (0.6,0.3)
\end{array}\right)
\end{aligned}
$$

This is not a null matrix.

This result also holds for both existing method and our method.

Here we have proved some properties of the proposed relational matrix obtained with the help of the proposed cartesian product on the basis of reference function and some numerical examples are presented to compare the proposed cartesian product of two fuzzy sets and the relational matrix obtained thereby with the existing methods. Numerical results show that the proposed method is similar to the existing one except in the case of representation of the elements of the relational matrix.

\section{Conclusions}

In this article, we have revisited the existing definitions of the cartesian product of two fuzzy matrices as well as the relational matrix associated with the cartesian product. Since there are some drawbacks in the existing definition of complementation of fuzzy sets so here we have worked towards removing those drawbacks with the use of reference function in defining fuzzy sets. The main contribution of this article is to define cartesian product and the fuzzy relational matrix in accordance with the reference function. It is expected that with the use of reference function, the drawbacks that currently exists can be removed to some extent.

\section{Acknowledgements}

The authors would like to thank the anonymous reviewers for their careful reading of this article and for their helpful comments.

\section{References}

[1] Zadeh L. A,Fuzzy Sets, Inform and Control, 1965,8: 338-353

[2] Zadeh L.A, Similarity Relations and Fuzzy orderings, Information Sciences, 3(1971), 177-200.

[3] Baruah H K, Fuzzy Membership with respect to a Reference Function, Journal of the Assam Science Society, 1999, 40(.3):65-73.

[4] Baruah H.K, Twards Forming a field of fuzzy sets, IJEIC, 2(1), 2011, 16-20

[5] Baruah H.K, Theory of Fuzzy sets Beliefs and Realities, IJEIC, 2 (2), 2011, 1-22Dhar. M, On Hwang and Yang's definition of Entropy of Fuzzy sets, IJLTC, UK, Vol.2, No.4, 2011, p-496-497.

[6] Dhar.M, Fuzzy Sets Towards Forming Boolean Algebra, IJEIC, Korea, Vol.2, Issue 4, 2011, p137-142.

[7] Dhar. M, A Note on existing Definition of Fuzzy Entropy, IJEIC, Korea, Vol.3, Issue 1, 2012, p-1721.

[8] Dhar.M, On Separation Index of Fuzzy Sets, IJMA, Vol.3, No.3, 2012, p-932-934.

[9] Dhar.M, On Geometrical Representation of Fuzzy Numbers, IJEIC, Korea, Vol.3, Issue 2, 2012, p29-34.

[10] Dhar.M, On Fuzzy Measures of Symmetry Breaking of Conditions, Similarity and Comparisons: Non Statistical Information for the Single Patient. IJMA, 2516-2519, 3(7), 2012. 
[11] Dhar.M, A Note on Subsethood measure of Fuzzy sets, IJEIC, Korea.,55-61, 3(3)

[12] Dhar.M, On Cardinality of Fuzzy sets, accepted for publication in IJISA

[13] Dhar. M, Representation of fuzzy matrices Based on Reference Function, I.J. Intelligence Systems and Applications, 2013, 5(2), 84-90.

[14] Dhar.M, A Note on Determinant and Adjoint of Fuzzy Square Matrix I.J. Intelligence Systems and Applications, 5(5), 58-67

\section{Author's Profiles}

Mamoni Dhar is an Assistant Professor in the department of Mathematics, Science College, Kokrajhar, Assam, India. She received M.Sc degree from Gauhati University, M.Phil degree from Madurai Kamraj University, B.Ed from Gauhati University and PGDIM from Indira Gandhi National Open University. Her research interest is in Fuzzy Mathematics. She has published eighteen articles in different national and international journals. 